\title{
Measurement of the mass of the W boson using direct reconstruction at $\sqrt{s}=183 \mathrm{GeV}$
}

DELPHI Collaboration

\begin{abstract}
From data corresponding to an integrated luminosity of $53.5 \mathrm{pb}^{-1}$ taken during the $183 \mathrm{GeV}$ run in 1997, DELPHI has measured the W mass from direct reconstruction of $\mathrm{WW} \rightarrow \ell \bar{\nu} \mathrm{q} \overline{\mathrm{q}}$ and $\mathrm{WW} \rightarrow \mathrm{q} \overline{\mathrm{q}} \mathrm{q} \overline{\mathrm{q}}$ events. Combining these channels, a value of $m_{\mathrm{W}}=80.238 \pm 0.154$ (stat) \pm 0.035 (syst) \pm 0.035 ( f si) \pm $0.021(L E P) \mathrm{GeV} / \mathrm{c}^{2}$ is obtained, where $f$ si denotes final state interaction.

Combined with the W mass obtained by DELPHI from the WW production cross-section and with the direct measurement at $172 \mathrm{GeV}$ this leads to a measured value of $m_{\mathrm{W}}=80.270 \pm 0.137$ (stat) \pm 0.031 (syst) $\pm 0.030(\mathrm{fsi}) \pm$ $0.021(L E P) \mathrm{GeV} / \mathrm{c}^{2}$, in good agreement with the Standard Model expectation. The width of the $\mathrm{W}$ boson is also measured, giving the value $\Gamma_{W}=2.48 \pm$ 0.40 (stat) \pm 0.10 (syst) $\mathrm{GeV} / \mathrm{c}^{2}$.
\end{abstract}


P.Abreu $^{21}$, T.Adye ${ }^{36}$, P.Adzic ${ }^{11}$, Z.Albrecht ${ }^{17}$, T.Alderweireld ${ }^{2}$, G.D.Alekseev ${ }^{16}$, R.Alemany ${ }^{49}$, T.Allmendinger ${ }^{17}$, P.P.Allport ${ }^{22}$, S.Almehed ${ }^{24}$, U.Amaldi ${ }^{9}$, N.Amapane ${ }^{45}$, S.Amato ${ }^{47}$, E.G.Anassontzis ${ }^{3}$, P.Andersson ${ }^{44}$, A.Andreazza ${ }^{9}$, S.Andringa ${ }^{21}$, P.Antilogus ${ }^{25}$, W-D.Apel ${ }^{17}$, Y.Arnoud ${ }^{9}$, B.Åsman ${ }^{44}$, J-E.Augustin ${ }^{25}$, A.Augustinus ${ }^{9}, \quad$ P.Baillon ${ }^{9}$, P.Bambade ${ }^{19}$, F.Barao ${ }^{21}$, G.Barbiellini ${ }^{46}$, R.Barbier ${ }^{25}$, D.Y.Bardin ${ }^{16}$, G.Barker ${ }^{17}$, A.Baroncelli ${ }^{38}$, M.Battaglia ${ }^{15}$, M.Baubillier ${ }^{23}$, K-H.Becks ${ }^{52}$, M.Begalli ${ }^{6}$, A.Behrmann ${ }^{52}$, P.Beilliere ${ }^{8}$, Yu.Belokopytov ${ }^{9,53}$, K.Belous ${ }^{42}$, N.C.Benekos ${ }^{31}$, A.C.Benvenuti ${ }^{5}$, C.Berat ${ }^{14}$, M.Berggren ${ }^{25}$, D.Bertini ${ }^{25}$, D.Bertrand ${ }^{2}, \quad$ M.Besancon ${ }^{39}$, M.Bigi ${ }^{45}$, M.S.Bilenky ${ }^{16}$, M-A.Bizouard ${ }^{19}$, D.Bloch ${ }^{10}, \quad$ H.M.Blom ${ }^{30}, \quad$ M.Bonesini ${ }^{27}, \quad$ W.Bonivento ${ }^{27}, \quad$ M.Boonekamp ${ }^{39}, \quad$ P.S.L.Booth ${ }^{22}$, A.W.Borgland ${ }^{4}$, G.Borisov ${ }^{19}$, C.Bosio ${ }^{41}$, O.Botner ${ }^{48}$, E.Boudinov ${ }^{30}$, B.Bouquet ${ }^{19}$, C.Bourdarios ${ }^{19}$, T.J.V.Bowcock ${ }^{22}$, I.Boyko $^{16}$, I.Bozovic ${ }^{11}$, M.Bozzo ${ }^{13}$, P.Branchini ${ }^{38}$, T.Brenke ${ }^{52}$, R.A.Brenner ${ }^{48}$, P.Bruckman ${ }^{18}$, J-M.Brunet ${ }^{8}$, L.Bugge ${ }^{32}$, T.Buran $^{32}$, T.Burgsmueller ${ }^{52}$, B.Buschbeck ${ }^{50}$, P.Buschmann ${ }^{52}$, S.Cabrera ${ }^{49}$, M.Caccia ${ }^{27}$, M.Calvi ${ }^{27}$, T.Camporesi ${ }^{9}$, V.Canale ${ }^{37}$, F.Carena ${ }^{9}$, L.Carroll ${ }^{22}$, C.Caso ${ }^{13}$, M.V.Castillo Gimenez ${ }^{49}$, A.Cattai ${ }^{9}$, F.R.Cavallo ${ }^{5}$, V.Chabaud ${ }^{9}$, M.Chapkin ${ }^{42}$, Ph.Charpentier ${ }^{9}$, L.Chaussard ${ }^{25}$, P.Checchia ${ }^{35}$, G.A.Chelkov ${ }^{16}$, R.Chierici ${ }^{45}$, P.Chliapnikov ${ }^{42}$, P.Chochula ${ }^{7}$, V.Chorowicz ${ }^{25}$, J.Chudoba ${ }^{29}$, K.Cieslik ${ }^{18}$, P.Collins ${ }^{9}$, R.Contri ${ }^{13}$, E.Cortina ${ }^{49}$, G.Cosme $^{19}$, F.Cossutti ${ }^{9}$, J-H.Cowell ${ }^{22}$, H.B.Crawley ${ }^{1}$, D.Crennell ${ }^{36}$, S.Crepe ${ }^{14}$, G.Crosetti ${ }^{13}$, J.Cuevas Maestro ${ }^{33}$, S.Czellar ${ }^{15}$, M.Davenport ${ }^{9}$, W.Da Silva ${ }^{23}$, A.Deghorain ${ }^{2}$, G.Della Ricca ${ }^{46}$, P.Delpierre ${ }^{26}$, N.Demaria ${ }^{9}$, A.De Angelis ${ }^{9}$, W.De Boer ${ }^{17}$, C.De Clercq ${ }^{2}$, B.De Lotto ${ }^{46}$, A.De Min ${ }^{35}$, L.De Paula ${ }^{47}$, H.Dijkstra ${ }^{9}$, L.Di Ciaccio ${ }^{37,9}$, J.Dolbeau ${ }^{8}$, K.Doroba ${ }^{51}$, M.Dracos ${ }^{10}$, J.Drees $^{52}$, M.Dris $^{31}$, A.Duperrin ${ }^{25}$, J-D.Durand ${ }^{9}$, G.Eigen ${ }^{4}$, T.Ekelof ${ }^{48}$, G.Ekspong ${ }^{44}$, M.Ellert ${ }^{48}$, M.Elsing ${ }^{9}$, J-P.Engel ${ }^{10}$, B.Erzen ${ }^{43}$, M.Espirito Santo ${ }^{21}$, G.Fanourakis ${ }^{11}$, D.Fassouliotis ${ }^{11}$, J.Fayot ${ }^{23}$, M.Feindt ${ }^{17}$, P.Ferrari ${ }^{27}$, A.Ferrer ${ }^{49}$, E.Ferrer-Ribas ${ }^{19}$, F.Ferro ${ }^{13}$, S.Fichet ${ }^{23}$, A.Firestone ${ }^{1}$, U.Flagmeyer ${ }^{52}$, H.Foeth ${ }^{9}$, E.Fokitis ${ }^{31}$, F.Fontanelli ${ }^{13}$, B.Franek ${ }^{36}$, A.G.Frodesen ${ }^{4}$, R.Fruhwirth ${ }^{50}$, F.Fulda-Quenzer ${ }^{19}$, J.Fuster ${ }^{49}$, A.Galloni ${ }^{22}$, D.Gamba ${ }^{45}$, S.Gamblin ${ }^{19}$, M.Gandelman ${ }^{47}$, C.Garcia $^{49}$, C.Gaspar ${ }^{9}$, M.Gaspar ${ }^{47}$, U.Gasparini ${ }^{35}$, Ph.Gavillet ${ }^{9}$, E.N.Gazis ${ }^{31}$, D.Gele ${ }^{10}$, N.Ghodbane ${ }^{25}$, I.Gil ${ }^{49}$, F.Glege $\mathrm{e}^{52}$, R.Gokieli ${ }^{9,51}$, B.Golob ${ }^{43}$, G.Gomez-Ceballos ${ }^{40}$, P.Goncalves ${ }^{21}$, I.Gonzalez Caballero ${ }^{40}, \quad$ G.Gopal ${ }^{36}$, L.Gorn ${ }^{1,54}$, Yu.Gouz ${ }^{42}$, V.Gracco ${ }^{13}$, J.Grahl ${ }^{1}$, E.Graziani ${ }^{38}$, C.Green ${ }^{22}$, H-J.Grimm ${ }^{17}$, P.Gris ${ }^{39}$, G.Grosdidier ${ }^{19}$, K.Grzelak ${ }^{51}$, M.Gunther ${ }^{48}$, J.Guy ${ }^{36}$, F.Hahn ${ }^{9}$, S.Hahn ${ }^{52}$, S.Haider ${ }^{9}$, A.Hallgren ${ }^{48}$, K.Hamacher ${ }^{52}$, J.Hansen ${ }^{32}$, F.J.Harris ${ }^{34}$, V.Hedberg ${ }^{24}$, S.Heising ${ }^{17}$, J.J.Hernandez ${ }^{49}$, P.Herquet ${ }^{2}$, H.Herr ${ }^{9}$, T.L.Hessing ${ }^{34}$, J.-M.Heuser ${ }^{52}$, E.Higon ${ }^{49}$, S-O.Holmgren ${ }^{44}$, P.J.Holt ${ }^{34}$, S.Hoorelbeke ${ }^{2}$, M.Houlden ${ }^{22}$, J.Hrubec ${ }^{50}$, K.Huet ${ }^{2}$, G.J.Hughes ${ }^{22}$, K.Hultqvist ${ }^{44}$, J.N.Jackson ${ }^{22}$, R.Jacobsson ${ }^{9}$, P.Jalocha ${ }^{9}$, R.Janik ${ }^{7}, \quad$ Ch.Jarlskog ${ }^{24}$ ， G.Jarlskog ${ }^{24}$, P.Jarry ${ }^{39}$, B.Jean-Marie ${ }^{19}$, E.K.Johansson ${ }^{44}$, P.Jonsson ${ }^{25}$, C.Joram ${ }^{9}$, P.Juillot ${ }^{10}$, F.Kapusta ${ }^{23}$, K.Karafasoulis ${ }^{11}$, S.Katsanevas ${ }^{25}$, E.C.Katsoufis ${ }^{31}$, R.Keranen ${ }^{17}$, B.P.Kersevan ${ }^{43}$, B.A.Khomenko ${ }^{16}$, N.N.Khovanski ${ }^{16}$, A.Kiiskinen ${ }^{15}$, B.King ${ }^{22}$, A.Kinvig ${ }^{22}$, N.J.Kjaer ${ }^{30}$, O.Klapp ${ }^{52}$, H.Klein ${ }^{9}$, P.Kluit ${ }^{30}$, P.Kokkinias ${ }^{11}$, M.Koratzinos ${ }^{9}$, V.Kostioukhine ${ }^{42}$, C.Kourkoumelis ${ }^{3}$, O.Kouznetsov ${ }^{39}$, M.Krammer ${ }^{50}$, E.Kriznic ${ }^{43}$, J.Krstic ${ }^{11}$, Z.Krumstein ${ }^{16}$, P.Kubinec ${ }^{7}$, J.Kurowska ${ }^{51}$, K.Kurvinen ${ }^{15}$, J.W.Lamsa ${ }^{1}$,

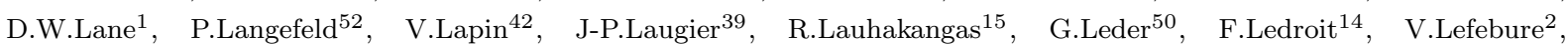
L.Leinonen $^{44}$, A.Leisos ${ }^{11}$, R.Leitner ${ }^{29}$, J.Lemonne ${ }^{2}$, G.Lenzen ${ }^{52}$, V.Lepeltier ${ }^{19}$, T.Lesiak ${ }^{18}$, M.Lethuillier ${ }^{39}$, J.Libby ${ }^{34}$, D.Liko $^{9}$, A.Lipniacka ${ }^{44}$, I.Lippi ${ }^{35}$, B.Loerstad ${ }^{24}$, J.G.Loken ${ }^{34}$, J.H.Lopes ${ }^{47}$, J.M.Lopez ${ }^{40}$, R.Lopez-Fernandez ${ }^{14}$, D.Loukas $^{11}$, P.Lutz ${ }^{39}$, L.Lyons ${ }^{34}$, J.MacNaughton ${ }^{50}$, J.R.Mahon ${ }^{6}$, A.Maio ${ }^{21}$, A.Malek ${ }^{52}$, T.G.M.Malmgren ${ }^{44}$,

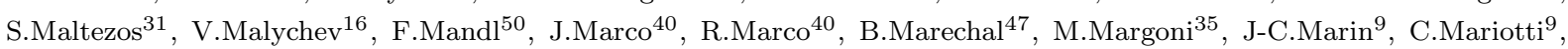
A.Markou $^{11}$, C.Martinez-Rivero ${ }^{19}, \quad$ F.Martinez-Vidal ${ }^{49}, \quad$ S.Marti i Garcia ${ }^{9}, \quad$ J.Masik $^{12}, \quad$ N.Mastroyiannopoulos ${ }^{11}$, F.Matorras ${ }^{40}$, C.Matteuzzi ${ }^{27}$, G.Matthiae ${ }^{37}$, F.Mazzucato ${ }^{35}$, M.Mazzucato ${ }^{35}$, M.Mc Cubbin ${ }^{22}, \quad$ R.Mc Kay ${ }^{1}$, R.Mc Nulty ${ }^{22}$, G.Mc Pherson ${ }^{22}$, C.Meroni ${ }^{27}$, W.T.Meyer ${ }^{1}$, E.Migliore ${ }^{45}$, L.Mirabito ${ }^{25}$, W.A.Mitaroff ${ }^{50}$, U.Mjoernmark ${ }^{24}$, T.Moa ${ }^{44}$, M.Moch ${ }^{17}$, R.Moeller ${ }^{28}$, K.Moenig ${ }^{9}$, M.R.Monge ${ }^{13}$, X.Moreau ${ }^{23}$, P.Morettini ${ }^{13}$, G.Morton ${ }^{34}$, U.Mueller ${ }^{52}$, K.Muenich ${ }^{52}$, M.Mulders ${ }^{30}$, C.Mulet-Marquis ${ }^{14}$, R.Muresan ${ }^{24}$, W.J.Murray ${ }^{36}$, B.Muryn ${ }^{14,18}$, G.Myatt ${ }^{34}$, T.Myklebust $^{32}$, F.Naraghi ${ }^{14}, \quad$ M.Nassiakou ${ }^{11}, \quad$ F.L.Navarria ${ }^{5}, \quad$ S.Navas ${ }^{49}, \quad$ K.Nawrocki $^{51}, \quad$ P.Negri ${ }^{27}, \quad$ S.Nemecek ${ }^{12}, \quad$ N.Neufeld $^{9}$, R.Nicolaidou ${ }^{39}$, B.S.Nielsen ${ }^{28}$, P.Niezurawski ${ }^{51}$, M.Nikolenko ${ }^{10,16}$, V.Nomokonov ${ }^{15}$, A.Normand ${ }^{22}$, A.Nygren $^{24}$, V.Obraztsov ${ }^{42}$, A.G.Olshevski ${ }^{16}$, A.Onofre ${ }^{21}$, R.Orava ${ }^{15}$, G.Orazi ${ }^{10}$, K.Osterberg ${ }^{15}$, A.Ouraou ${ }^{39}$, M.Paganoni ${ }^{27}$, S.Paiano $^{5}$, R.Pain ${ }^{23}$, R.Paiva ${ }^{21}$, J.Palacios ${ }^{34}$, H.Palka ${ }^{18}$, Th.D.Papadopoulou ${ }^{31,9}$, K.Papageorgiou ${ }^{11}$, L.Pape ${ }^{9}$, C.Parkes $^{9}$, F.Parodi ${ }^{13}$, U.Parzefall ${ }^{22}$, A.Passeri ${ }^{38}$, O.Passon ${ }^{52}$, M.Pegoraro ${ }^{35}$, L.Peralta ${ }^{21}$, M.Pernicka ${ }^{50}$, A.Perrotta ${ }^{5}$, C.Petridou $^{46}$, A.Petrolini ${ }^{13}$, H.T.Phillips ${ }^{36}$, F.Pierre ${ }^{39}$, M.Pimenta ${ }^{21}$, E.Piotto ${ }^{27}$, T.Podobnik ${ }^{43}$, M.E.Pol ${ }^{6}$, G.Polok ${ }^{18}$, P.Poropat ${ }^{46}$, V.Pozdniakov $^{16}$, P.Privitera ${ }^{37}$, N.Pukhaeva ${ }^{16}$, A.Pullia ${ }^{27}$, D.Radojicic ${ }^{34}$, S.Ragazzi ${ }^{27}$, H.Rahmani ${ }^{31}$, P.N.Ratoff ${ }^{20}$, A.L.Read ${ }^{32}$, P.Rebecchi ${ }^{9}$, N.G.Redaelli ${ }^{27}, \quad$ M.Regler ${ }^{50}$, D.Reid ${ }^{30}$, R.Reinhardt ${ }^{52}$, P.B.Renton ${ }^{34}, \quad$ L.K.Resvanis ${ }^{3}$, F.Richard ${ }^{19}$, J.Ridky ${ }^{12}$, G.Rinaudo ${ }^{45}$, O.Rohne ${ }^{32}$, A.Romero ${ }^{45}, \quad$ P.Ronchese ${ }^{35}$, E.I.Rosenberg ${ }^{1}, \quad$ P.Rosinsky ${ }^{7}$, P.Roudeau ${ }^{19}$, T.Rovelli ${ }^{5}$, Ch.Royon ${ }^{39}$, V.Ruhlmann-Kleider ${ }^{39}$, A.Ruiz ${ }^{40}$, H.Saarikko ${ }^{15}$, Y.Sacquin ${ }^{39}$, A.Sadovsky ${ }^{16}$, G.Sajot $^{14}$, J.Salt ${ }^{49}$, D.Sampsonidis ${ }^{11}$, M.Sannino ${ }^{13}$, H.Schneider ${ }^{17}$, Ph.Schwemling ${ }^{23}$, B.Schwering ${ }^{52}$, U.Schwickerath ${ }^{17}$, M.A.E.Schyns ${ }^{52}$, F.Scuri ${ }^{46}$, P.Seager ${ }^{20}$, Y.Sedykh ${ }^{16}$, A.M.Segar ${ }^{34}$, R.Sekulin ${ }^{36}$, R.C.Shellard ${ }^{6}$, A.Sheridan ${ }^{22}$, M.Siebel $^{52}$, L.Simard ${ }^{39}$, F.Simonetto ${ }^{35}$, A.N.Sisakian ${ }^{16}$, G.Smadja ${ }^{25}$, O.Smirnova ${ }^{24}$, G.R.Smith ${ }^{36}$, A.Sokolov ${ }^{42}$, O.Solovianov ${ }^{42}$,

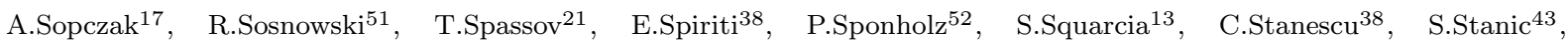
K.Stevenson ${ }^{34}$, A.Stocchi ${ }^{19}$, J.Strauss ${ }^{50}$, R.Strub ${ }^{10}$, B.Stugu ${ }^{4}$, M.Szczekowski ${ }^{51}$, M.Szeptycka ${ }^{51}$, T.Tabarelli ${ }^{27}$, F.Tegenfeldt ${ }^{48}$, F.Terranova ${ }^{27}$, J.Thomas ${ }^{34}$, J.Timmermans ${ }^{30}$, N.Tinti ${ }^{5}$, L.G.Tkatchev ${ }^{16}$, S.Todorova ${ }^{10}$, A.Tomaradze ${ }^{2}$, B.Tome ${ }^{21}$, A.Tonazzo ${ }^{9}$, L.Tortora ${ }^{38}$, G.Transtromer ${ }^{24}$, D.Treille ${ }^{9}$, G.Tristram ${ }^{8}$, M.Trochimczuk ${ }^{51}$, C.Troncon ${ }^{27}$, 
A.Tsirou ${ }^{9}$, M-L.Turluer ${ }^{39}$, I.A.Tyapkin ${ }^{16}$, S.Tzamarias ${ }^{11}, \quad$ O.Ullaland ${ }^{9}, \quad$ V.Uvarov ${ }^{42}$, G.Valenti ${ }^{5}, \quad$ E.Vallazza ${ }^{46}$, C.Vander Velde ${ }^{2}$, G.W.Van Apeldoorn ${ }^{30}$, P.Van Dam ${ }^{30}$, W.K.Van Doninck ${ }^{2}$, J.Van Eldik ${ }^{30}$, A.Van Lysebetten ${ }^{2}$, N.Van Remortel ${ }^{2}$, I.Van Vulpen ${ }^{30}$, N.Vassilopoulos ${ }^{34}$, G.Vegni ${ }^{27}$, L.Ventura ${ }^{35}$, W.Venus ${ }^{36,9}$, F.Verbeure ${ }^{2}$, M.Verlato ${ }^{35}$, L.S.Vertogradov ${ }^{16}$, V.Verzi ${ }^{37}$, D.Vilanova ${ }^{39}$, L.Vitale ${ }^{46}$, E.Vlasov ${ }^{42}$, A.S.Vodopyanov ${ }^{16}$, C.Vollmer ${ }^{17}$, G.Voulgaris ${ }^{3}$, V.Vrba $^{12}$, H.Wahlen ${ }^{52}$, C.Walck ${ }^{44}$, C.Weiser ${ }^{17}$, D.Wicke ${ }^{52}$, J.H.Wickens ${ }^{2}$, G.R.Wilkinson ${ }^{9}$, M.Winter ${ }^{10}$, M.Witek $^{18}$, G.Wolf ${ }^{9}$, J.Yi ${ }^{1}, \quad$ O.Yushchenko ${ }^{42}, \quad$ A.Zaitsev ${ }^{42}, \quad$ A.Zalewska ${ }^{18}, \quad$ P.Zalewski ${ }^{51}, \quad$ D.Zavrtanik ${ }^{43}$, E.Zevgolatakos ${ }^{11}$, N.I.Zimin ${ }^{16,24}$, G.C.Zucchelli ${ }^{44}$, G.Zumerle ${ }^{35}$

${ }^{1}$ Department of Physics and Astronomy, Iowa State University, Ames IA 50011-3160, USA

${ }^{2}$ Physics Department, Univ. Instelling Antwerpen, Universiteitsplein 1, BE-2610 Wilrijk, Belgium and IIHE, ULB-VUB, Pleinlaan 2, BE-1050 Brussels, Belgium

and Faculté des Sciences, Univ. de l'Etat Mons, Av. Maistriau 19, BE-7000 Mons, Belgium

${ }^{3}$ Physics Laboratory, University of Athens, Solonos Str. 104, GR-10680 Athens, Greece

${ }^{4}$ Department of Physics, University of Bergen, Allégaten 55, NO-5007 Bergen, Norway

${ }^{5}$ Dipartimento di Fisica, Università di Bologna and INFN, Via Irnerio 46, IT-40126 Bologna, Italy

${ }^{6}$ Centro Brasileiro de Pesquisas Físicas, rua Xavier Sigaud 150, BR-22290 Rio de Janeiro, Brazil and Depto. de Física, Pont. Univ. Católica, C.P. 38071 BR-22453 Rio de Janeiro, Brazil

and Inst. de Física, Univ. Estadual do Rio de Janeiro, rua São Francisco Xavier 524, Rio de Janeiro, Brazil

${ }^{7}$ Comenius University, Faculty of Mathematics and Physics, Mlynska Dolina, SK-84215 Bratislava, Slovakia

${ }^{8}$ Collège de France, Lab. de Physique Corpusculaire, IN2P3-CNRS, FR-75231 Paris Cedex 05, France

${ }^{9} \mathrm{CERN}, \mathrm{CH}-1211$ Geneva 23, Switzerland

${ }^{10}$ Institut de Recherches Subatomiques, IN2P3 - CNRS/ULP - BP20, FR-67037 Strasbourg Cedex, France

${ }^{11}$ Institute of Nuclear Physics, N.C.S.R. Demokritos, P.O. Box 60228, GR-15310 Athens, Greece

${ }^{12}$ FZU, Inst. of Phys. of the C.A.S. High Energy Physics Division, Na Slovance 2, CZ-180 40, Praha 8, Czech Republic

${ }^{13}$ Dipartimento di Fisica, Università di Genova and INFN, Via Dodecaneso 33, IT-16146 Genova, Italy

${ }^{14}$ Institut des Sciences Nucléaires, IN2P3-CNRS, Université de Grenoble 1, FR-38026 Grenoble Cedex, France

${ }^{15}$ Helsinki Institute of Physics, HIP, P.O. Box 9, FI-00014 Helsinki, Finland

${ }^{16}$ Joint Institute for Nuclear Research, Dubna, Head Post Office, P.O. Box 79, RU-101 000 Moscow, Russian Federation

${ }^{17}$ Institut für Experimentelle Kernphysik, Universität Karlsruhe, Postfach 6980, DE-76128 Karlsruhe, Germany

${ }^{18}$ Institute of Nuclear Physics and University of Mining and Metalurgy, Ul. Kawiory 26a, PL-30055 Krakow, Poland

${ }^{19}$ Université de Paris-Sud, Lab. de l'Accélérateur Linéaire, IN2P3-CNRS, Bât. 200, FR-91405 Orsay Cedex, France

${ }^{20}$ School of Physics and Chemistry, University of Lancaster, Lancaster LA1 4YB, UK

${ }^{21}$ LIP, IST, FCUL - Av. Elias Garcia, 14-1 ${ }^{\circ}$, PT-1000 Lisboa Codex, Portugal

${ }^{22}$ Department of Physics, University of Liverpool, P.O. Box 147, Liverpool L69 3BX, UK

${ }^{23}$ LPNHE, IN2P3-CNRS, Univ. Paris VI et VII, Tour 33 (RdC), 4 place Jussieu, FR-75252 Paris Cedex 05, France

${ }^{24}$ Department of Physics, University of Lund, Sölvegatan 14, SE-223 63 Lund, Sweden

${ }^{25}$ Université Claude Bernard de Lyon, IPNL, IN2P3-CNRS, FR-69622 Villeurbanne Cedex, France

${ }^{26}$ Univ. d'Aix - Marseille II - CPP, IN2P3-CNRS, FR-13288 Marseille Cedex 09, France

${ }^{27}$ Dipartimento di Fisica, Università di Milano and INFN, Via Celoria 16, IT-20133 Milan, Italy

${ }^{28}$ Niels Bohr Institute, Blegdamsvej 17, DK-2100 Copenhagen Ø, Denmark

${ }^{29}$ NC, Nuclear Centre of MFF, Charles University, Areal MFF, V Holesovickach 2, CZ-180 00, Praha 8, Czech Republic

${ }^{30}$ NIKHEF, Postbus 41882, NL-1009 DB Amsterdam, The Netherlands

${ }^{31}$ National Technical University, Physics Department, Zografou Campus, GR-15773 Athens, Greece

${ }^{32}$ Physics Department, University of Oslo, Blindern, NO-1000 Oslo 3, Norway

${ }^{33}$ Dpto. Fisica, Univ. Oviedo, Avda. Calvo Sotelo s/n, ES-33007 Oviedo, Spain

${ }^{34}$ Department of Physics, University of Oxford, Keble Road, Oxford OX1 3RH, UK

${ }^{35}$ Dipartimento di Fisica, Università di Padova and INFN, Via Marzolo 8, IT-35131 Padua, Italy

${ }^{36}$ Rutherford Appleton Laboratory, Chilton, Didcot OX11 OQX, UK

${ }^{37}$ Dipartimento di Fisica, Università di Roma II and INFN, Tor Vergata, IT-00173 Rome, Italy

${ }^{38}$ Dipartimento di Fisica, Università di Roma III and INFN, Via della Vasca Navale 84, IT-00146 Rome, Italy

${ }^{39}$ DAPNIA/Service de Physique des Particules, CEA-Saclay, FR-91191 Gif-sur-Yvette Cedex, France

${ }^{40}$ Instituto de Fisica de Cantabria (CSIC-UC), Avda. los Castros s/n, ES-39006 Santander, Spain

${ }^{41}$ Dipartimento di Fisica, Università degli Studi di Roma La Sapienza, Piazzale Aldo Moro 2, IT-00185 Rome, Italy

${ }^{42}$ Inst. for High Energy Physics, Serpukov P.O. Box 35, Protvino, (Moscow Region), Russian Federation

${ }^{43}$ J. Stefan Institute, Jamova 39, SI-1000 Ljubljana, Slovenia and Laboratory for Astroparticle Physics,

Nova Gorica Polytechnic, Kostanjeviska 16a, SI-5000 Nova Gorica, Slovenia,

and Department of Physics, University of Ljubljana, SI-1000 Ljubljana, Slovenia

${ }^{44}$ Fysikum, Stockholm University, Box 6730, SE-113 85 Stockholm, Sweden

${ }^{45}$ Dipartimento di Fisica Sperimentale, Università di Torino and INFN, Via P. Giuria 1, IT-10125 Turin, Italy

${ }^{46}$ Dipartimento di Fisica, Università di Trieste and INFN, Via A. Valerio 2, IT-34127 Trieste, Italy

and Istituto di Fisica, Università di Udine, IT-33100 Udine, Italy

${ }^{47}$ Univ. Federal do Rio de Janeiro, C.P. 68528 Cidade Univ., Ilha do Fundão BR-21945-970 Rio de Janeiro, Brazil

${ }^{48}$ Department of Radiation Sciences, University of Uppsala, P.O. Box 535, SE-751 21 Uppsala, Sweden

${ }^{49}$ IFIC, Valencia-CSIC, and D.F.A.M.N., U. de Valencia, Avda. Dr. Moliner 50, ES-46100 Burjassot (Valencia), Spain

${ }^{50}$ Institut für Hochenergiephysik, Österr. Akad. d. Wissensch., Nikolsdorfergasse 18, AT-1050 Vienna, Austria

${ }^{51}$ Inst. Nuclear Studies and University of Warsaw, Ul. Hoza 69, PL-00681 Warsaw, Poland

${ }^{52}$ Fachbereich Physik, University of Wuppertal, Postfach 100 127, DE-42097 Wuppertal, Germany

${ }^{53} \mathrm{On}$ leave of absence from IHEP Serpukhov

${ }^{54}$ Now at University of Florida 


\section{Introduction}

During the $183 \mathrm{GeV}$ run in 1997 DELPHI collected a sample of $\mathrm{e}^{+} \mathrm{e}^{-}$collisions corresponding to an integrated luminosity of $53.5 \mathrm{pb}^{-1}$. At this energy a high precision measurement of $m_{\mathrm{W}}$ can be made by reconstructing the $\mathrm{W}$ mass spectrum using constrained fitting techniques. This direct measurement of $m_{\mathrm{W}}$ provides an important test of the Standard Model by comparison with the indirect measurement from precise electroweak results at lower energies [1] and helps constraining the mass of the Higgs boson.

This paper describes the analysis using events in which one $\mathrm{W}$ decays into leptons and the other into quarks, WW $\rightarrow \ell \bar{\nu} \mathrm{q} \overline{\mathrm{q}}$ ("semileptonic" events), and events in which both Ws decay hadronically, WW $\rightarrow$ q $\bar{q} q \bar{q}$ ("hadronic" events).

The paper is organised as follows. In section 2, the DELPHI detector setup and the event generators are briefly reviewed. In section 3 , the measurement of the $\mathrm{W}$ mass in the semileptonic channel is presented, while section 4 describes this measurement in the fully hadronic channel. In section 5 , the measurement of the width of the $\mathrm{W}$ in both channels is described. In section 6 , the combined results for the mass and the width are presented, as well as combinations with previous DELPHI results.

\section{Apparatus and Simulations} [2].

Detailed descriptions of the DELPHI apparatus and its performance can be found in

The response of the detector to various physics processes was modelled using the simulation program DELSIM [3], which incorporates the resolution, granularity and efficiency of the detector components. The event generator EXCALIBUR [4] was used for the simulation of all four-fermion final states (signal and background), while the background from $\mathrm{e}^{+} \mathrm{e}^{-} \rightarrow \mathrm{q} \overline{\mathrm{q}}(\gamma)$ was generated with the PYTHIA [5] event generator. For the generation of the EXCALIBUR events, the fragmentation was performed using JETSET 7.4 [6] tuned to the DELPHI LEP1 data [7], and the initial state radiation (ISR) using the QEDPS program [8]. Systematic checks were performed using other generators as described in the relevant sections.

For the signal part, a sample of 210,000 events was generated with a reference $\mathrm{W}$ mass of $80.35 \mathrm{GeV} / \mathrm{c}^{2}$, while two other smaller samples of 120,000 events each were generated with masses of 79.35 and $81.35 \mathrm{GeV} / \mathrm{c}^{2}$. At each of these masses, the simulated width was the one predicted by the standard model. The $\mathrm{W}$ mass and width used in this paper correspond to a $\mathrm{W}$ propagator with an s-dependent width.

\section{Analysis of the semileptonic decay channel}

Events were selected from the data sample recorded, requiring all detectors essential for this measurement to be fully efficient. These comprise the central tracking detectors and the electromagnetic calorimeters. The recorded sample corresponds to an integrated luminosity of $52.0 \mathrm{pb}^{-1}$.

\subsection{Track selection, lepton identification and event selection}

The selection of semileptonic candidates was optimized to keep events where the lepton is a muon or an electron. 
Charged particles were selected if they fulfilled the following criteria :

- momentum greater than $0.2 \mathrm{GeV} / \mathrm{c}$;

- impact parameter with respect to the nominal interaction point less than $4 \mathrm{~cm}$ (transverse) and $10 \mathrm{~cm}$ (longitudinal).

For neutral particles, at least one of the following selection requirements was to be fulfilled :

- energy of the shower in the electromagnetic calorimeter greater than $0.2 \mathrm{GeV}$;

- energy of the shower in the hadron calorimeter greater than $0.5 \mathrm{GeV}$.

Charged particles were identified as muons if they were associated with a hit in the muon chambers or an energy deposition in the hadron calorimeter consistent with a minimum ionizing particle. Muon identification was performed in the polar angle range between $10^{\circ}$ and $170^{\circ}$ where the identification efficiency was determined from simulation to be $(92 \pm 1) \%$.

Electron identification was performed in the polar angle range between $20^{\circ}$ and $160^{\circ}$ by looking for charged particles with a characteristic energy deposition in the electromagnetic calorimeters. The energy was required to be within $30 \%$ of the measured track momentum or to exceed $20 \mathrm{GeV}$. In order to increase the efficiency of the selection in the forward/backward regions (below $36^{\circ}$ and above $144^{\circ}$ ), where the momentum resolution is poorer, tracks with electromagnetic energy above $8 \mathrm{GeV}$ and negligible hadron calorimeter energy deposition were considered as electrons. Within the whole accepted region, the identification efficiency was determined from simulation to be $(77 \pm 2) \%$.

The events were selected with the same criteria as for the analysis at $172 \mathrm{GeV}[9]$ except for the following :

- All neutral deposits in a cone of $3^{\circ}$ around the muon candidate were rejected from the analysis.

- All electromagnetic clusters, not associated to a charged particle track, in a cone of $10^{\circ}$ around the electron candidate were excluded from the jet clustering and their electromagnetic energy added to that of the electron.

- The isolation of the lepton candidate was enhanced :

- the angle between the lepton and the nearest jet axis had to be at least $20^{\circ}$;

- the sum of the charged and of the neutral energy depositions in a cone between $10^{\circ}$ and $15^{\circ}$ around the electron candidate had to be less than $1 \mathrm{GeV}$.

After the selection, 88 electron and 109 muon candidates remained in the data. The number of expected events from simulation is 80.2 with a purity of $91.0 \%$ in the electron channel and 100.7 with a purity of $94.5 \%$ in the muon channel.

\subsection{Kinematic reconstruction}

The events were reconstructed using a constrained fit, imposing equality of the two W masses, as described in [9]. In contrast to the previous analysis, however, events were not forced into a two-jet configuration : the LUCLUS algorithm [6] (with djoin $=7.5 \mathrm{GeV} / \mathrm{c}$ ) was used to reconstruct jets once the lepton candidate had been taken out. Events with more than three jets were then reclustered, forcing them to a three-jet configuration.

The distribution of the reconstructed masses is shown in figure 1 for real and simulated data in the electron and muon channels. 
DELPHI

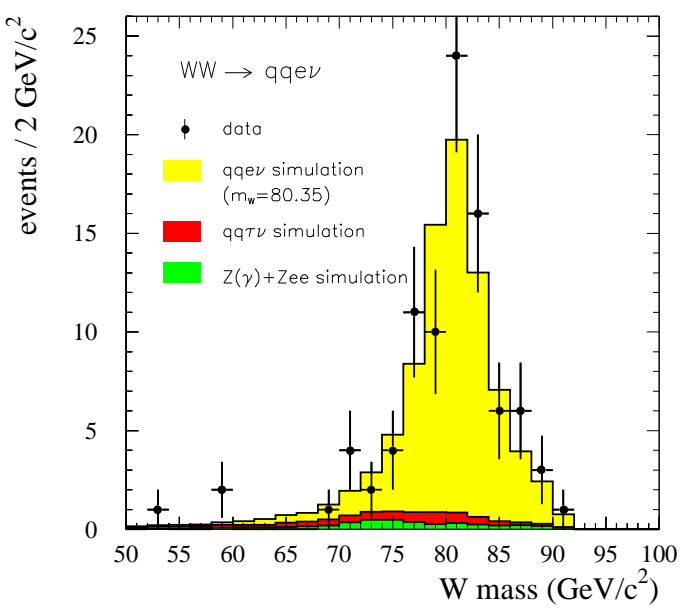

DELPHI

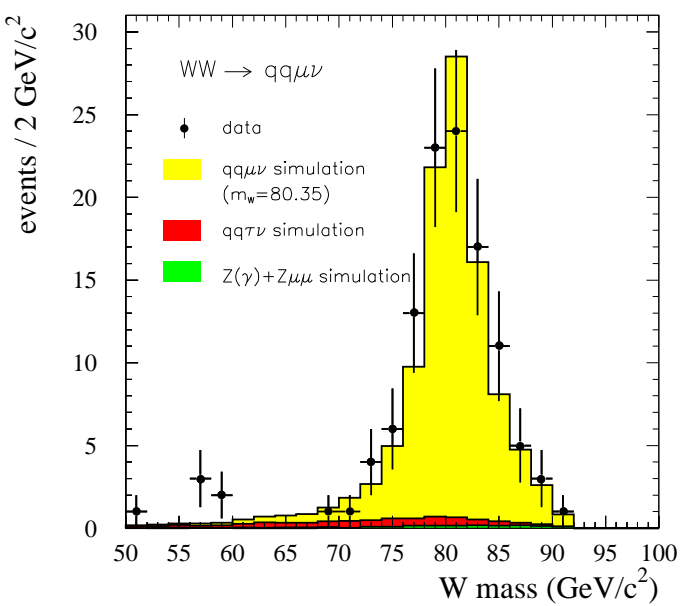

Figure 1: The distributions of the reconstructed masses for the electron and muon channels

\subsection{Fitting method}

The W mass was extracted from the reconstructed mass distribution using the method already used in the analysis of the data at $172 \mathrm{GeV}$ [9], i.e. an event-by-event maximum likelihood fit to a relativistic Breit-Wigner convoluted with a Gaussian resolution function plus a background distribution. The shape of the background was taken from the simulation. The error on the reconstructed mass from the constrained kinematic fit was used as the width of the Gaussian for the corresponding event. Only events in the mass range between 69 and $91 \mathrm{GeV} / \mathrm{c}^{2}$ were used in the fit.
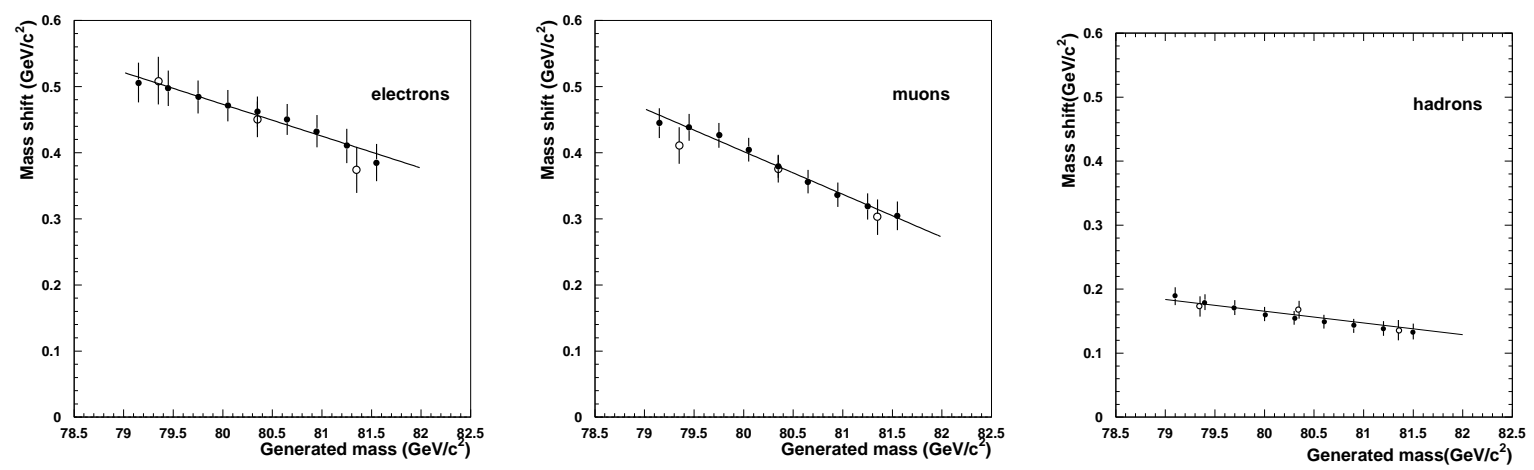

Figure 2: Calibration curves for the mass shift (fitted mass minus generated mass) from $\mathrm{q} \overline{\mathrm{q}} \mathrm{\nu} \nu$ (left), $\mathrm{q} \overline{\mathrm{q}} \mu \nu$ (centre) and $\mathrm{q} \overline{\mathrm{q}} \mathrm{q} \overline{\mathrm{q}}$ (right) events. The open circles show the mass shift computed from the individual simulated samples, while the full circles are determined from the full statistics reweighted to the corresponding mass. The error bars in the reweighted case are completely correlated and indicate the statistical error on the mass shift for a given true mass.

The bias from this method (shown in fig 2) comes mainly from the initial state radiation which is not properly taken into account in the fit, and was estimated from simulated events either generated at various input values of $m_{W}$ or using a reweighting technique in 
order to obtain events for arbitrary values of $m_{W}$. The bias is well described by a straight line which is used to correct the result of the likelihood fit. The error on the bias, coming from the limited statistics of the simulation, is given by the statistical error corresponding to all samples of simulated events reweighted at the measured mass. The negative slope (fig 2) is mainly due to ISR and reduces slightly the sensitivity of the fitted mass to the true one. The statistical error coming from the mass fit is increased accordingly.

Samples of simulated signal and background events corresponding to the integrated luminosity of the data were processed through the same mass reconstruction and fitting procedures as the real data. The pulls $\left(m_{r e c}-m_{\text {gen }}\right) / \sigma\left(m_{r e c}\right)$ obtained with these samples were used to check that the statistical error from the likelihood fit was reliable. The RMS of the pull distributions is equal to one in the electron and muon channels, with a mean expected error of $418 \mathrm{MeV} / \mathrm{c}^{2}$ for the electrons and $331 \mathrm{MeV} / \mathrm{c}^{2}$ for the muons.

\subsection{Systematic errors}

The analysis described above relies on a bias correction obtained from the simulation. Any error on the simulation will then cause a systematic error on the mass. The different sources of systematic errors are discussed in detail in [9]. The list of the relevant ones is presented in table 1.

\begin{tabular}{|l|cc|c|c|c|}
\hline $\begin{array}{c}\text { Sources of systematic error } \\
\left(\mathrm{MeV} / \mathrm{c}^{2}\right)\end{array}$ & $\mathrm{e} \bar{\nu} \mathrm{q} \overline{\mathrm{q}}$ & $\mu \bar{\nu} \mathrm{q} \overline{\mathrm{q}}$ & $\mathrm{l} \overline{\mathrm{q}} \overline{\mathrm{q}}$ & $\mathrm{q} \overline{\mathrm{q}} \mathrm{q} \overline{\mathrm{q}}$ & Combined \\
\hline \hline Statistical error on calibration & 23 & 18 & 14 & 9 & 8 \\
Lepton energy & 40 & 35 & 26 & - & 9 \\
Jet energy & 50 & 30 & 38 & 20 & 26 \\
Background level & 5 & - & 2 & 5 & 3 \\
Background shape & - & - & - & 5 & 3 \\
Isolation of the lepton & 20 & - & 8 & - & 3 \\
\hline Total uncorrelated & $\mathbf{7 1}$ & $\mathbf{5 0}$ & $\mathbf{4 9}$ & $\mathbf{2 3}$ & $\mathbf{2 9}$ \\
\hline \hline Fragmentation & 10 & 10 & 10 & 20 & 17 \\
I.S.R. & 10 & 10 & 10 & 10 & 10 \\
\hline Total correlated & $\mathbf{1 4}$ & $\mathbf{1 4}$ & $\mathbf{1 4}$ & $\mathbf{2 2}$ & $\mathbf{2 0}$ \\
\hline \hline LEP energy & $\mathbf{2 1}$ & $\mathbf{2 1}$ & $\mathbf{2 1}$ & $\mathbf{2 1}$ & $\mathbf{2 1}$ \\
\hline \hline Colour reconnection & - & - & - & 50 & 33 \\
Bose Einstein correlations & - & - & - & 20 & 13 \\
\hline Total final state interaction & - & - & - & $\mathbf{5 4}$ & $\mathbf{3 5}$ \\
\hline
\end{tabular}

Table 1: Contributions to the systematic error on the mass measurement. The error sources have been separated into those uncorrelated and correlated between the different LEP experiments.

The statistical errors on the bias correction coming from the limited simulation statistics are 23 and $18 \mathrm{MeV} / \mathrm{c}^{2}$, respectively, for the electron and muon channels.

Due to the presence of the neutrino in this channel, the dominant systematic effects are due to the uncertainty on the absolute energy calibrations. Bhabha and Compton scattering events showed an uncertainty on the electron energy of $1 \%$, while the systematic uncertainty on the muon momentum was estimated from $\mathrm{Z}^{0} \rightarrow \mu^{+} \mu^{-}$events to be $0.5 \%$. This corresponds to uncorrelated systematic errors of 40 and $35 \mathrm{MeV} / \mathrm{c}^{2}$, respectively. 
Jet energy uncertainty was estimated to be $2 \%$ from $\mathrm{Z}^{0} \rightarrow q \bar{q}$ events, using two-jet events where the energy is fixed and three-jet events where the energy can be estimated from angular measurement alone. The resulting systematic errors are $50 \mathrm{MeV} / \mathrm{c}^{2}$ for the electron events and $30 \mathrm{MeV} / \mathrm{c}^{2}$ for the muon events. The different sensitivity on the energy calibrations for the two lepton types arises because the constrained kinematic fit uses the error on the lepton energy, which is smaller for the muons than for electrons.

The impact of the background was very small in these channels, and a change of $10 \%$ in the background level led to a $5 \mathrm{MeV} / \mathrm{c}^{2}$ change on the mass for the electrons, and a negligible one for the muons.

In order to simulate the effect of an imperfect description of the lepton acceptance in the simulation (which could induce a bias on the mass), the cut at $1 \mathrm{GeV}$ on the neutral energy deposition in a cone between 5 and $15^{\circ}$ was varied by $0.5 \mathrm{GeV}$ on the simulation only and the fit was repeated. The observed shift $\left(20 \mathrm{MeV} / \mathrm{c}^{2}\right)$ was taken as the systematic error from this source.

The uncertainty coming from the modelling of the initial state radiation in the simulation was estimated by comparing the distribution of the energy lost by ISR in events simulated with our EXCALIBUR generator and in events simulated with the KORALW generator [10]. The agreement was found to be very good, and an uncertainty of 10 $\mathrm{MeV} / \mathrm{c}^{2}$ was derived.

When fitting the mass of the $\mathrm{W}$, the width of the Breit-Wigner was fixed. The chosen value has no importance as it led to negligible changes on the fitted mass, and is anyway corrected for by the calibration curve. What is more relevant here is the value of the width which has been used for the generation of the simulated events. The simulated events have been reweighted in order to reproduce a shift of one standard deviation on the world average measurement of the width $\left(60 \mathrm{MeV} / \mathrm{c}^{2}[11]\right)$ and the whole procedure was repeated. The resulting effect on the measured mass was found to be negligible.

For the lepton combination, all sources of systematic errors are taken as uncorrelated between electron and muon, except for the errors coming from the jet energy, the fragmentation and the ISR which are taken as fully correlated.

\subsection{Results}

The event-by-event likelihood analysis on the semileptonic channels gave the following results:

$$
m_{W}=80.612 \pm 0.431(\text { stat }) \pm 0.072(\text { syst }) \pm 0.021(\text { LEP }) \mathrm{GeV} / \mathrm{c}^{2}
$$

for electron events and

$$
m_{W}=80.462 \pm 0.341(\text { stat }) \pm 0.052(\text { syst }) \pm 0.021(\text { LEP }) \mathrm{GeV} / \mathrm{c}^{2}
$$

for muon events. The last error ${ }^{1}$ comes from the uncertainty on the beam energy (25 MeV [12]).

The combination of the two gives the following result:

$$
m_{W}=80.520 \pm 0.267(\text { stat }) \pm 0.051 \text { (syst) } \pm 0.021(\text { LEP }) \mathrm{GeV} / \mathrm{c}^{2}
$$

\footnotetext{
${ }^{1} \Delta m_{\mathrm{W}} / m_{\mathrm{W}} \approx \Delta \mathrm{E}_{\mathrm{beam}} / \mathrm{E}_{\mathrm{beam}}$
} 


\section{Analysis of the hadronic decay channel}

The present analysis is largely based on the analysis used by DELPHI to extract the $\mathrm{W}$ mass from the $172 \mathrm{GeV}$ data [9]. It is also an event-by-event likelihood method, taking into account all jet-pairings. However, in contrast to the analysis of [9], the masses of the two $\mathrm{W}$ bosons are not assumed to be equal. Instead, the $\mathrm{W}$ mass is extracted using a two-dimensional ideogram likelihood method.

Requiring only that the central tracking was fully efficient, events were selected from a data sample corresponding to an integrated luminosity of $52.9 \mathrm{pb}^{-1}$.

\subsection{Event selection}

In the fully hadronic channel, emphasis was put on obtaining a high efficiency for $\mathrm{WW} \rightarrow \mathrm{q} \overline{\mathrm{q}} \mathrm{q} \overline{\mathrm{q}}$ events that contain useful information about the $\mathrm{W}$ mass. Obtaining a high purity in the selected sample was considered to have a lower priority, because the background from two-fermion processes is taken into account by an estimated event-byevent purity later in the analysis, and the contribution from hadronic ZZ events is included as a separate term in the likelihood expression (see section 4.3). Instead the selection was designed to be minimally biased by the mass information contained in the event. The following selection criteria were used:

- Events were required to have at least 14 reconstructed tracks;

- A visible energy of more than $1.15 E_{\text {beam }}$ was required;

- At least four jets had to be reconstructed using the Durham [13] algorithm with $y_{\text {cut }}=0.001$

- On forcing the event into a four-jet configuration, each jet had to have at least three particles and an invariant mass larger than $1 \mathrm{GeV} / \mathrm{c}^{2}$;

- The invariant mass of the whole jet system, excluding isolated high energy photons inside the detector and estimated by doing a constrained kinematic fit assuming that there was one (additional) photon of unknown energy radiated into the beampipe, had to be larger than $150 \mathrm{GeV} / \mathrm{c}^{2}$.

The efficiency and purity (with respect to WW events) of this selection were estimated to be $88 \%$ and $66 \%$ respectively. A total of 540 events were selected from the data (see figure 3). The number of events expected from simulation was 518 .

\subsection{Kinematic reconstruction}

A constrained fit [9] was used to obtain optimal precision on the 4-momenta of the jets.

In the fully hadronic channel there are three possible jet-pairings when there are four jets, and 10 in a five-jet event. Events with more than five jets were forced into a five-jet configuration. For every possible jet-pairing, the probability density function $\mathrm{p}_{\mathrm{i}}\left(m_{x}, m_{y}\right)$ that this pairing corresponds to two objects with masses $m_{x}$ and $m_{y}$ was computed : a fit with constraints from energy and momentum conservation was performed, fixing the two masses to $m_{x}$ and $m_{y}$, and the probability $\mathrm{p}_{\mathrm{i}}$ was derived from the resulting $\chi^{2}$ as $\mathrm{p}_{\mathrm{i}}\left(m_{x}, m_{y}\right) \propto \exp \left(-\frac{1}{2} \chi_{i}^{2}\left(m_{x}, m_{y}\right)\right)$.

The different jet-pairings are equivalent from the point of view of goodness-of-fit. However, jet-pairings that have a small difference between the two fitted masses will naturally have a larger impact on the $\mathrm{W}$ mass likelihood (see section 4.3), due to the limited decay 
DELPHI

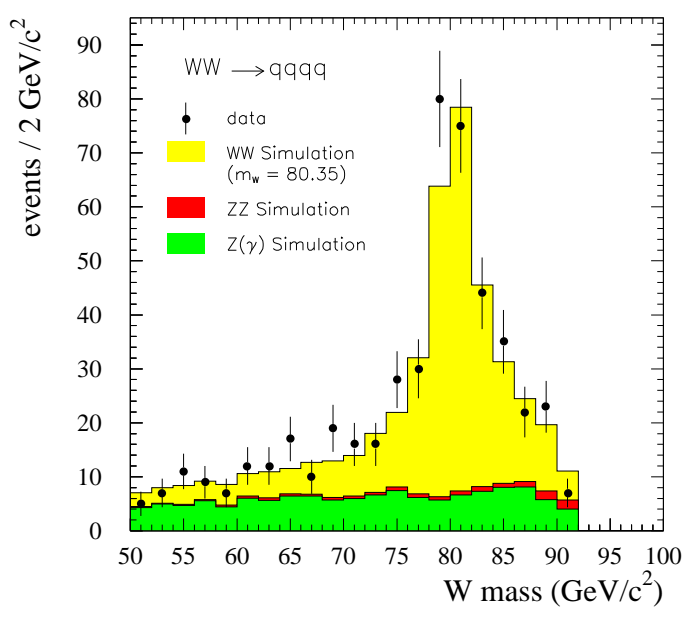

Figure 3: Mass plot for the selected $\mathrm{WW} \rightarrow \mathrm{q} \overline{\mathrm{q}} \mathrm{q} \overline{\mathrm{q}}$ candidates showing only one reconstructed mass per event (that with the best $\chi^{2}$ ), and using an equal-mass constraint (not used in the determination of $m_{W}$, see text).

width of the $\mathrm{W}$ boson. In order to improve the jet-pairing further without biasing the mass, relative probabilities were calculated that a jet-pairing is the correct one. This was done in the following way:

- The measured jet charges $^{2}$ were used to determine the probability for a proposed $\mathrm{W}$ boson to be a $\mathrm{W}^{+}$or a $\mathrm{W}^{-}$. From this probability, a relative weight was calculated corresponding to the production angle, assuming a flat distribution for the combinatorial background.

- For 5-jet events, the most probable gluon jet candidate in every jet-pairing was chosen from the 3 jets supposed to belong to one $\mathrm{W}$ boson by taking the jet with the lowest transverse momentum, $k_{\mathrm{t}}$, with respect to the other two jets in the rest-frame of the $\mathrm{W}$ boson. The relative weight for that jet-pairing was then multiplied by the probability $p \propto \frac{1}{k_{\mathrm{t}}}$ to emit a gluon with the observed transverse momentum.

Using these weights, the $\mathrm{p}_{\mathrm{i}}\left(m_{x}, m_{y}\right)$ distributions were added in a weighted sum. Thus for every event a probability density function was obtained containing all the extracted mass information from the kinematics of the event. This so-called two-dimensional probability ideogram reflects the relative compatibility of the kinematics of the event with the hypothesis that two heavy objects with the corresponding masses $m_{x}, m_{y}$ were produced, with a $\frac{1}{k_{\mathrm{t}}}$ gluon emission spectrum and a production angle distributed like in $\mathrm{W}^{+} \mathrm{W}^{-}$ events. Examples are shown in figure 4.

In order to reduce the contribution from events with poorly reconstructed jets, this procedure was repeated with two other jet algorithms (CAMJET [14] and DICLUS [15]). The number of jets was fixed to the number found with the DURHAM algorithm. The three ideograms were then simply added with equal weights. Events with ambiguous clustering give combined ideograms with broader resolutions. This allowed us to reduce

\footnotetext{
${ }^{2}$ The jet charges were computed as the weighted charge of particles in the jet, with a weight proportional to the square root of the longitudinal momentum.
} 

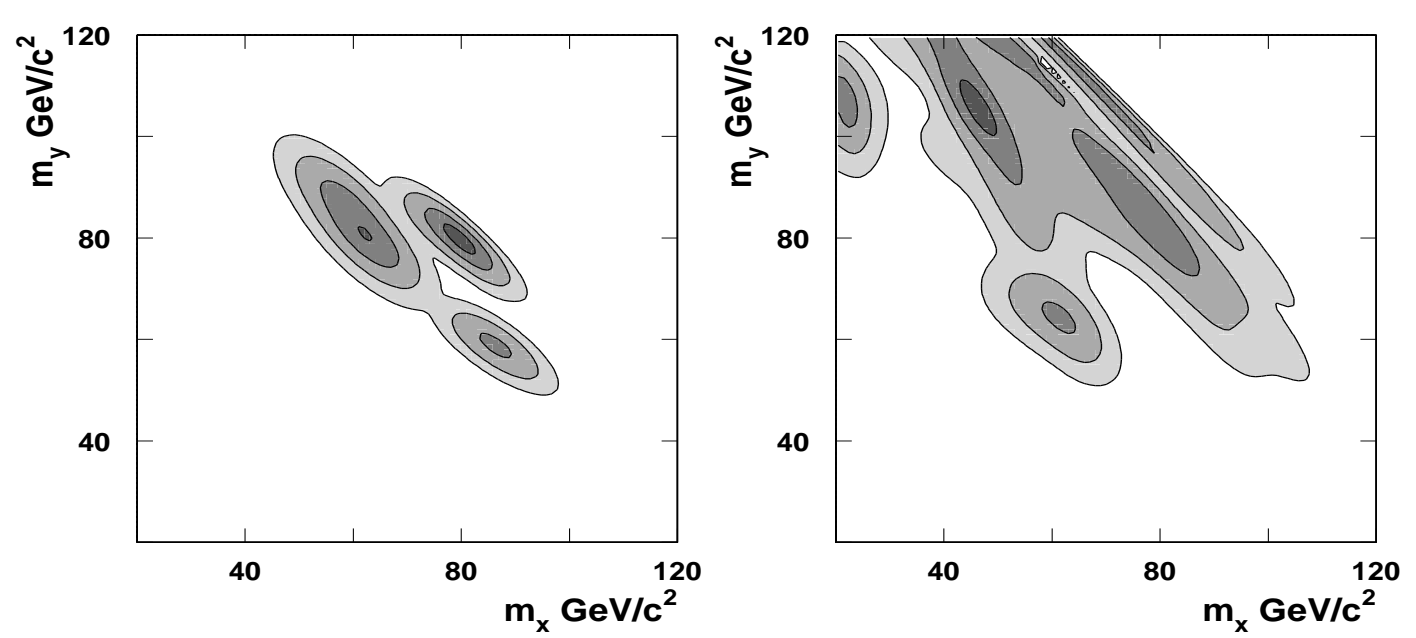

Figure 4: Examples of two-dimensional probability ideograms for a 4-jet (left) and 5-jet (right) hadronic event. The first 4 sigma contours are shown.

the overall jet errors in the constrained fit by $20 \%$. This procedure reduced the expected statistical error on $m_{\mathrm{W}}$ by $4 \%$.

\subsection{Event-by-event likelihood}

For every event the likelihood $\mathcal{L}_{e}\left(m_{\mathrm{W}}, \Gamma_{\mathrm{W}}\right)$ of the $\mathrm{W}$ mass and width was calculated using

$$
\begin{aligned}
& \mathcal{L}_{e}\left(m_{\mathrm{W}}, \Gamma_{\mathrm{W}}\right)= \\
& \qquad \int \sum_{i} p_{i, e}\left(m_{x}, m_{y}\right)\left\{P_{e}^{e f f} \cdot \mathrm{S}\left(m_{x}, m_{y}, m_{\mathrm{W}}, \Gamma_{\mathrm{W}}\right)+\left(1-P_{e}^{e f f}\right) \cdot \mathrm{B}\left(m_{x}, m_{y}\right)\right\} d m_{x} d m_{y}
\end{aligned}
$$

where $\mathrm{S}\left(m_{x}, m_{y}, m_{\mathrm{W}}, \Gamma_{\mathrm{W}}\right)$ is the probability that a mass combination $m_{x}, m_{y}$ is produced in a 4-fermion event, $\mathrm{B}\left(m_{x}, m_{y}\right)$ the corresponding distribution for the background processes, and $P_{e}^{e f f}$ the estimated effective purity of the event, i.e. the probability that the event is a well-clustered fully hadronic 4-fermion event. The significant contributions to $\mathrm{S}\left(m_{x}, m_{y}, m_{\mathrm{W}}, \Gamma_{\mathrm{W}}\right)$ are from resonant $\mathrm{WW}$ and ZZ production; all other contributions, including the interference between these two resonant processes, were found to be negligible. These two contributions were weighted according to their accepted cross-sections:

$$
\begin{aligned}
& \mathrm{S}\left(m_{x}, m_{y}, m_{\mathrm{W}}, \Gamma_{\mathrm{W}}\right)= \\
& \quad \frac{\sigma_{\mathrm{WW}}^{\mathrm{acc}}}{\sigma_{\mathrm{WW}}^{\mathrm{acc}}+\sigma_{\mathrm{ZZ}}^{\mathrm{acc}}} \cdot \mathrm{S}_{\mathrm{WW}}\left(m_{x}, m_{y}, m_{\mathrm{W}}, \Gamma_{\mathrm{W}}\right)+\frac{\sigma_{\mathrm{ZZ}}^{\mathrm{acc}}}{\sigma_{\mathrm{WW}}^{\mathrm{acc}}+\sigma_{\mathrm{ZZ}}^{\mathrm{acc}}} \cdot \mathrm{S}_{\mathrm{ZZ}}\left(m_{x}, m_{y}, m_{\mathrm{Z}}, \Gamma_{\mathrm{Z}}\right)
\end{aligned}
$$

where $\mathrm{S}_{\mathrm{WW}}\left(m_{x}, m_{y}, m_{\mathrm{W}}, \Gamma_{\mathrm{W}}\right)$ and $\mathrm{S}_{\mathrm{ZZ}}\left(m_{x}, m_{y}, m_{\mathrm{Z}}, \Gamma_{\mathrm{Z}}\right)$ were approximated by the product of two relativistic Breit-Wigner functions and a phase-space correction factor $\operatorname{PS}\left(m_{x}, m_{y}\right)$

$$
\operatorname{PS}\left(m_{x}, m_{y}\right) \propto \frac{\sqrt{\left(s-m_{x}^{2}-m_{y}^{2}\right)^{2}-4 m_{x}^{2} m_{y}^{2}}}{s}
$$

and both $\mathrm{S}_{\mathrm{WW}}\left(m_{x}, m_{y}, m_{\mathrm{W}}, \Gamma_{\mathrm{W}}\right)$ and $\mathrm{S}_{\mathrm{ZZ}}\left(m_{x}, m_{y}, m_{\mathrm{Z}}, \Gamma_{\mathrm{Z}}\right)$ were normalized to one over the integration area. The background function $\mathrm{B}\left(m_{x}, m_{y}\right)$ was taken to be proportional 


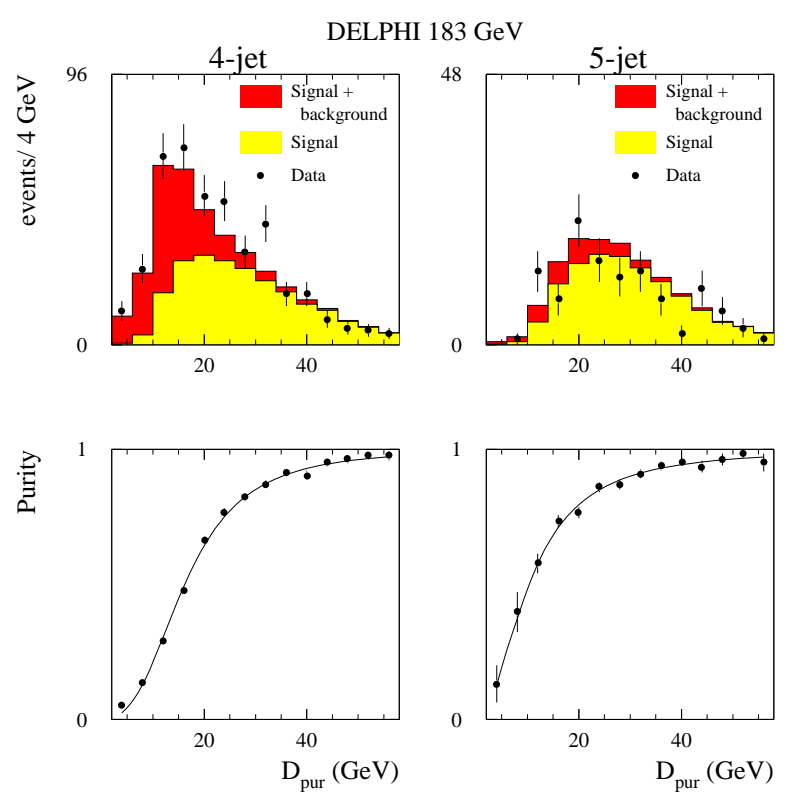

Figure 5: Parameterisation of the purity per event : the top plots show the distribution of $D_{\text {pur }}=E_{\min } \theta_{\min }$ for data and simulated events in four-jet (left) and five-jet (right) configurations, after applying all event selection cuts; the bottom plots show the parametrization of the purity per event as a function of $D_{\text {pur }}$ obtained from the simulated events.

to the same phase-space correction function $\operatorname{PS}\left(m_{x}, m_{y}\right)$. Monte Carlo simulation shows that this is a good approximation.

To obtain the effective event purity $P_{e}^{e f f}$, the signal-to-(signal+background) ratio was parametrized as a function of a discriminating variable $D_{\text {pur }}=\theta_{\min } \cdot E_{\min }$, where $\theta_{\min }$ is the smallest angle between any two jets, and $E_{\min }$ the lowest jet energy (see figure 5 ). In this calculation fully hadronic ZZ events are treated as signal and $\mathrm{Z} \gamma$ events as background. This purity was multiplied by a factor $\epsilon_{\text {cluster }}$, estimating the fraction of the events in which the clustering algorithm succeeds in separating the jets correctly. Both in the four-jet and five-jet cases, $\epsilon_{\text {cluster }}(=0.80)$ was determined from a global tuning where the jet errors (in the kinematic fit) and $\epsilon_{\text {cluster }}$ were adjusted so that the width of the pull distribution (see end of this section) should be equal to unity and the bias on the $\mathrm{W}$ width determination (see section 5) minimized. This efficiency was cross-checked with simulated events from the distribution of the quantity $\chi^{2}\left(m_{x}^{\text {gen }}, m_{y}^{\text {gen }}\right)-\chi_{\min }^{2}\left(m_{x}, m_{y}\right)$ where $\chi^{2}\left(m_{x}^{\text {gen }}, m_{y}^{\text {gen }}\right)$ and $\chi_{\min }^{2}\left(m_{x}, m_{y}\right)$ are obtained, respectively, from kinematic fits with $m_{x}$ and $m_{y}$ at their generated values and at the values giving the best fit to the event. This quantity is expected to follow a $\chi^{2}$ distribution with two degrees of freedom, but, in practice, $20 \%$ of events are found to lie in a tail with too high values.

As the candidate events are not correlated, the combined likelihood is the product of all the event likelihoods.

The bias from this method (shown in figure 2) as a function of the true $\mathrm{W}$ mass was determined using the three simulated samples with different input values for the generated $\mathrm{W}$ mass. As in section 3.3, a linear dependence was fitted and used to correct the result of the likelihood fit.

In order to check if the statistical error obtained from the likelihood curve is a good estimator, pull distributions were studied using one million samples of simulated events. 
The samples were composed from simulated events generated with $m_{\mathrm{W}}=80.35 \mathrm{GeV} / \mathrm{c}^{2}$ and according to Poissonian statistics corresponding to the expected number of signal and background events. The mean RMS of the pull distribution as a function of the estimated error is compatible with unity within $2 \%$, which means that the error obtained from the likelihood curve is a reliable estimate of the statistical error on the $\mathrm{W}$ mass. The mean expected error was $193 \mathrm{MeV} / \mathrm{c}^{2}$.

\subsection{Systematic errors}

The calibration of the analysis depends on the accuracy of the simulation. Errors in the simulation can lead to systematic shifts of the measured W mass. Table 1 shows the estimated systematic errors coming from possible inaccuracies in the simulation.

The statistical error on the bias correction, coming from the limited simulation statistics, is $9 \mathrm{MeV} / \mathrm{c}^{2}$. The uncertainty coming from the jet energy calibration is much reduced compared to the semileptonic case because of the absence of missing energy. It corresponds to a $20 \mathrm{MeV} / \mathrm{c}^{2}$ error. The systematic error coming from the background level has been estimated by changing its amount by $10 \%$, while the uncertainty from the shape of the background mass distribution has been taken from the difference between the two Monte Carlo generators JETSET [6] and ARIADNE [16]. Both lead to a $5 \mathrm{MeV} / \mathrm{c}^{2}$ systematic error. The impact of an incorrect description of the jet fragmentation has been studied by using WW events simulated with two different treatments of the parton shower (JETSET and ARIADNE). The difference was compatible with zero with a $20 \mathrm{MeV} / \mathrm{c}^{2}$ statistical error which was taken as the systematic error from this source.

Since the two Ws under LEP2 conditions decay much closer to each other than the typical hadronization scale of $0.5-1.0 \mathrm{fm}$, interactions among the decay products in fully hadronic events may lead to systematic shifts of the reconstructed $\mathrm{W}$ mass. Two possible sources of such effects have been identified: colour reconnection among partons from the two different colour singlet systems [17] and Bose-Einstein correlations among identical bosons in the final state [18]. Colour reconnection during the perturbative phase of the parton shower evolution has been shown to give rise to mass shifts below $5 \mathrm{MeV} / \mathrm{c}^{2}$ and is neglected here. Colour reconnection in the non-perturbative phase has been described in a number of phenomenological models, which are implemented in several Monte Carlo generators $[6,16,19]$. Whereas the expected error on the $\mathrm{W}$ mass from a first round of comparisons of models [20] was estimated to be around $50 \mathrm{MeV} / \mathrm{c}^{2}$, more recent studies [21] indicate that with the present mass reconstruction methods, the effect may be much smaller.

The effect was tested using two variants of the ARIADNE model. In the first variant (AR2), only gluons with energies less than $\Gamma_{W}$ are allowed to reconnect, whereas the second variant (AR3) allows also reconnections of gluons with higher energy. Since colour reconnection in the perturbative phase is expected to be suppressed [17], the latter model is theoretically disfavoured and will not be used in the estimate of the systematic error on

the $\mathrm{W}$ mass. The fast DELPHI detector simulation program [22] was used together with the event selection and mass reconstruction procedure above. The observed mass shifts were $28 \pm 6 \mathrm{MeV} / \mathrm{c}^{2}$ for $\mathrm{AR} 2$ and $55 \pm 6 \mathrm{MeV} / \mathrm{c}^{2}$ for AR3. Since other viable models of colour reconnection have not yet been investigated in studies specific to the present experiment, a systematic error in the $\mathrm{W}$ mass of $50 \mathrm{MeV} / \mathrm{c}^{2}$ is conservatively assigned for this effect.

The effect of Bose-Einstein correlations on the measured W mass has also been the subject of a number of recent phenomenological studies [23-26], which indicate that the 
shift is likely to be at most $20-30 \mathrm{MeV} / \mathrm{c}^{2}$ and possibly even smaller [27]. For the present paper this was tested in two detector specific studies in which the same event selection and mass reconstruction method as for the real data were used.

The first study was based on one of the global event weight schemes $[24]^{3}$ and used the EXCALIBUR Monte Carlo sample with $m_{\mathrm{W}}=80.35 \mathrm{GeV} / \mathrm{c}^{2}$ described above. In the second study a Bose-Einstein simulation code [27] was used, which is based on a modification of the JETSET fragmentation to include quantum mechanical interference effects among two or three identical bosons. The resulting shifts of the reconstructed $\mathrm{W}$ mass were $-10 \pm 10 \mathrm{MeV} / \mathrm{c}^{2}$ and $0 \pm 10 \mathrm{MeV} / \mathrm{c}^{2}$, respectively. The models were not retuned, however, and a systematic error on the $\mathrm{W}$ mass of $20 \mathrm{MeV} / \mathrm{c}^{2}$ due to BoseEinstein correlations is assigned.

The combined systematic error on the $\mathrm{W}$ mass measured in the fully hadronic channel from final state interactions is thus estimated to be at most $54 \mathrm{MeV} / \mathrm{c}^{2}$.

\subsection{Result for the hadronic channel}

The two-dimensional ideogram analysis on the hadronic channel gave the following result:

$$
m_{\mathrm{W}}=80.097 \pm 0.189(\text { stat }) \pm 0.032(\text { syst }) \pm 0.054(\text { fsi }) \pm 0.021(\text { LEP }),
$$

where ' $f s i$ ' denotes the possible effect from final state interactions and ' $L E P$ ' the uncertainty on the beam energy.

\subsection{Cross-check by an independent algorithm}

The W-boson mass was also measured in the fully hadronic channel by a different and independent method. A neural network was used to tag the signal, leading to an efficiency and purity of $85 \%$ and $80 \%$ respectively. The number of events selected from the data was 401, while 398 events were expected from studies performed on simulated events. In the mass reconstruction procedure, the jet multiplicity was left free to vary between four and eight jets. A fast kinematic fit was applied to improve the mass resolution of multijet events by imposing momentum and energy conservation while the directions of the jets were left unchanged. Events with more than five jets were forced into a five-jet configuration for both the kinematic fit and jet pairing stage.

The jet assignment was also performed using a neural network algorithm. For each combination, the interjet angles and the difference between the two reconstructed masses discriminate between the different clustering solutions. To reduce the bias arising from a single generated mass, the neural network was trained with $\mathrm{W}$ boson masses uniformly spread in the range $75<\mathrm{m}_{\mathrm{W}}<86 \mathrm{GeV} / \mathrm{c}^{2}$.

The $\mathrm{W}$ boson mass was extracted from a likelihood fit to the two-dimensional plot formed by the average and the difference of the two W-masses, using the distribution predicted by the full simulation. In order to obtain the simulated spectrum for arbitrary values of $\mathrm{m}_{\mathrm{W}}$, a Monte Carlo reweighting technique was used as in section 3.3. A binned log-likelihood fit to the data was then performed which avoids the analytical parametrization of the shapes. As the final states with jet multiplicities of four, five, and above have very different mass resolutions, the overall likelihood was the sum of three independent likelihoods, evaluated with the two-dimensional Monte Carlo probabilities relevant to each class of final states. The mass value and the statistical error obtained from this

\footnotetext{
${ }^{3}$ The weight function $V_{3}$ in [24] was used.
} 
likelihood fit to the data are $\mathrm{m}_{\mathrm{W}}=80.126 \pm 0.183 \mathrm{GeV} / \mathrm{c}^{2}$, in good agreement with the results quoted in section 4.5. The expected statistical error from the simulated events is $215 \mathrm{MeV} / \mathrm{c}^{2}$ and has been checked with samples of the same statistics as the data, using the technique described in section 4.3. The systematic uncertainties are common to the previous analysis, except for that due to the background shape. It is estimated to be around $15 \mathrm{MeV} / \mathrm{c}^{2}$ with an alternative fragmentation scheme (HERWIG instead of JETSET). The event-by-event likelihood analysis has been taken as the reference analysis because it gave the smaller expected error.

\section{Width of the $\mathrm{W}$ boson}

The same unbinned likelihood analyses (described in section 3.3 for the semileptonic channel and in section 4.3 for the hadronic one) were applied in order to measure the width of the $\mathrm{W}$ boson, fixing the mass and varying the width in the likelihood fit. A linear calibration curve was also used to correct the result from the fits. For this calibration curve, the Monte Carlo events have been reweighted to the mass of $80.35 \mathrm{GeV} / \mathrm{c}^{2}$, for different values of the width. The correlation between $m_{\mathrm{W}}$ and $\Gamma_{W}$ was found to have a negligible impact.

The centre-of-mass energy spread has been measured [12] and is about $220 \mathrm{MeV}$. Its impact on the width measurement is negligible. The dominant sources of systematic errors are presented in table 2 .

\begin{tabular}{|l|cc|c|c|c|}
\hline $\begin{array}{l}\text { Sources of systematic error } \\
\left(\mathrm{MeV} / \mathrm{c}^{2}\right)\end{array}$ & $\mathrm{e} \bar{\nu} \mathrm{q} \overline{\mathrm{q}}$ & $\mu \bar{\nu} \mathrm{q} \overline{\mathrm{q}}$ & $\mathrm{l} \bar{\nu} \mathrm{q} \overline{\mathrm{q}}$ & $\mathrm{q} \overline{\mathrm{q}} \overline{\mathrm{q}}$ & Combined \\
\hline \hline Statistical error on calibration & 56 & 54 & 39 & 32 & 26 \\
Lepton energy & 40 & 40 & 28 & - & 8 \\
Jet energy & 20 & 10 & 15 & 30 & 26 \\
Background & 40 & 20 & 22 & 60 & 44 \\
I.S.R. & 15 & 15 & 15 & 15 & 15 \\
Colour reconnection & - & - & - & 100 & 72 \\
Bose-Einstein correlation & - & - & - & 50 & 36 \\
\hline Total & $\mathbf{8 3}$ & $\mathbf{7 2}$ & $\mathbf{5 7}$ & $\mathbf{1 3 5}$ & $\mathbf{1 0 0}$ \\
\hline
\end{tabular}

Table 2: Dominant contributions to the systematic error on $\Gamma_{W}$.

For the semileptonic channels, the results are

$$
\begin{array}{lll}
\Gamma_{W}=2.09 \pm 1.10(\text { stat }) \pm 0.08(\text { syst }) \mathrm{GeV} / \mathrm{c}^{2} & \text { for electrons } \\
\Gamma_{W}=3.61 \pm 1.05(\text { stat }) \pm 0.07(\text { syst }) \mathrm{GeV} / \mathrm{c}^{2} & \text { for muons }
\end{array}
$$

which give, when combined,

$$
\Gamma_{W}=2.89 \pm 0.76(\text { stat }) \pm 0.06(\text { syst }) \mathrm{GeV} / \mathrm{c}^{2},
$$

while the hadronic channel gives

$$
\Gamma_{W}=2.33 \pm 0.47(\text { stat }) \pm 0.14(\text { syst }) \mathrm{GeV} / \mathrm{c}^{2} .
$$




\section{Combination of all results}

The masses measured in the semileptonic and hadronic decays analysis are in good agreement within statistics. Combining them yields

$$
m_{\mathrm{W}}=80.238 \pm 0.154(\text { stat }) \pm 0.035(\text { syst }) \pm 0.035(\text { f si }) \pm 0.021(\text { LEP }) \mathrm{GeV} / \mathrm{c}^{2} .
$$

Our previous measurements derived from the cross-section [28] $\left(m_{\mathrm{W}}=80.49 \pm 0.43(\right.$ stat $) \pm$ 0.09 (syst) $\left.\pm 0.03(L E P) \mathrm{GeV} / \mathrm{c}^{2}\right)$ and from our $172 \mathrm{GeV}$ data $[9]\left(m_{\mathrm{W}}=80.22 \pm\right.$ 0.41 (stat) \pm 0.04 (syst) $\left.\pm 0.03(f s i) \pm 0.03(L E P) \mathrm{GeV} / \mathrm{c}^{2}\right)^{4}$ are fully compatible with this more precise value. Combining all these measurements of the $\mathrm{W}$ mass yields

$$
m_{\mathrm{W}}=80.270 \pm 0.137 \text { (stat) } \pm 0.031 \text { (syst) } \pm 0.030(\text { fsi }) \pm 0.021(\text { LEP }) \mathrm{GeV} / \mathrm{c}^{2} .
$$

The width of the $\mathrm{W}$ has been measured. Combining results from the semileptonic and hadronic decay modes yields

$$
\Gamma_{W}=2.48 \pm 0.40(\text { stat }) \pm 0.10(\text { syst }) \mathrm{GeV} / \mathrm{c}^{2} .
$$

\section{Acknowledgements}

We are greatly indebted to our technical collaborators, to the members of the CERNSL Division for the excellent performance of the LEP collider, and to the funding agencies for their support in building and operating the DELPHI detector.

We acknowledge in particular the support of

Austrian Federal Ministry of Science and Traffics, GZ 616.364/2-III/2a/98,

FNRS-FWO, Belgium,

FINEP, CNPq, CAPES, FUJB and FAPERJ, Brazil,

Czech Ministry of Industry and Trade, GA CR 202/96/0450 and GA AVCR A1010521,

Danish Natural Research Council,

Commission of the European Communities (DG XII),

Direction des Sciences de la Matière, CEA, France,

Bundesministerium für Bildung, Wissenschaft, Forschung und Technologie, Germany,

General Secretariat for Research and Technology, Greece,

National Science Foundation (NWO) and Foundation for Research on Matter (FOM),

The Netherlands,

Norwegian Research Council,

State Committee for Scientific Research, Poland, 2P03B06015, 2P03B1116 and SPUB/P03/178/98,

JNICT-Junta Nacional de Investigação Científica e Tecnológica, Portugal,

Vedecka grantova agentura MS SR, Slovakia, Nr. 95/5195/134,

Ministry of Science and Technology of the Republic of Slovenia,

CICYT, Spain, AEN96-1661 and AEN96-1681,

The Swedish Natural Science Research Council,

Particle Physics and Astronomy Research Council, UK,

Department of Energy, USA, DE-FG02-94ER40817.

\footnotetext{
${ }^{4}$ Our present estimate of the fsi error has been applied to the $172 \mathrm{GeV}$ data.
} 


\section{References}

[1] LEP electroweak working group, A Combination of Preliminary Electroweak Measurements and Constraints on the Standard Model, CERN-EP/99-15.

[2] DELPHI Collaboration, P. Abreu et al., Nucl. Instr. Meth. A 303 (1991) 233;

DELPHI Collaboration, P. Abreu et al., Nucl. Instr. Meth. A 378 (1996) 57.

[3] DELPHI Collaboration, DELPHI event generation and detector simulation - User Guide, DELPHI 89-67.

[4] F. A. Berends, R. Pittau and R. Kleiss, Comp. Phys. Comm. 85 (1995) 437.

[5] T. Sjöstrand, Comp. Phys. Comm. 82 (1994) 74.

[6] T. Sjöstrand, PYTHIA 5.7/JETSET 7.4, CERN-TH.7112/93 (1993).

[7] DELPHI Collaboration, P. Abreu et al., Z. Phys. C 73 (1996) 11.

[8] T. Munehisa, J. Fujimoto, Y. Kurihara and Y. Shimizu, Prog. Theor. Phys. 95 (1996) 375.

[9] DELPHI Collaboration, P. Abreu et al., Eur. Phys. J. C 2 (1998) 581.

[10] M. Skrzypek, S. Jadach, W. Placzek and Z. Was, Comp. Phys. Comm. 94 (1996) 216.

[11] C. Caso et al., The 1998 Review of Particle Physics, Eur. Phys. J. C 3 (1998) 1.

[12] LEP energy working group, Evaluation of the LEP centre-of-mass energy above the W-pair production threshold, CERN-EP/98-191, submitted to Eur. Phys. J. C.

[13] S. Catani et al., Phys. Lett. B269 (1991) 432.

N. Brown and W.J. Stirling, Z. Phys. C 53 (1992) 629.

[14] Yu.L. Dokshitzer, G. Leder, S. Moretti and B. Webber, JHEP 08 (1997) 001.

[15] L. Lönnblad, Z. Phys. C 58 (1993) 471.

[16] L. Lönnblad, Comp. Phys. Comm. 71 (1992) 15.

[17] T. Sjöstrand and V. A. Khoze, Z. Phys. C 62 (1994) 281.

[18] L. Lönnblad and T. Sjöstrand, Phys. Lett. B 351 (1995) 293.

[19] G. Marchesini et al., Comp. Phys. Comm. 67 (1992) 465.

[20] Physics at LEP2, CERN Yellow report 96-01.

[21] S. Todorova-Nova, Mesure de la masse des bosons $W^{ \pm}$au LEP à l'aide du détecteur DELPHI, Thèse, Université Louis Pasteur de Strasbourg, IReS 98-18 (also available in http://home.cern.ch/ braize/thesis/todorova/todorova.ps.gz).

[22] DELPHI Collaboration, Fast simulation, DELPHI 87-26 PROG 71.

[23] S. Jadach and K. Zalewski, Acta Physica Polonica B 28 (1997) 1363.

[24] V. Kartvelishvili, R. Kvatadze and R. Møller, Phys. Lett. B 408 (1997) 331.

[25] K. Fialkowski and R. Wit, Acta Physica Polonica B 28 (1997) 2039.

K. Fialkowski and R. Wit, Eur. Phys. J. C 2 (1998) 691.

[26] L. Lönnblad and T. Sjöstrand, Eur. Phys. J. C 2 (1998) 165.

[27] S. Todorova and J. Rames, IReS 97/29, PRA-HEP97/16 and hep-ph/9710280.

[28] DELPHI Collaboration, P. Abreu et al., Phys. Lett. B 397 (1997) 158.

DELPHI Collaboration, P. Abreu et al., $W$ pair production cross-section and $W$ branching fractions in $e^{+} e^{-}$interactions at $183 \mathrm{GeV}$, CERN-EP/99-47, submitted to Phys. Lett. B 